\title{
SUPPLEMENTAL MATERIALS FOR "ESTIMATION AND INFERENCE IN GENERALIZED ADDITIVE COEFFICIENT MODELS FOR NONLINEAR INTERACTIONS WITH HIGH-DIMENSIONAL COVARIATES"
}

\author{
By Shujie Ma, ${ }^{*}$ Raymond J. Carroll ${ }^{\dagger}$ Hua \\ LIANG,$\ddagger$ AND SHIZHONG XU \\ University of California at Riverside, Texas A\&M University, George \\ Washington University, and University of California at Riverside \\ In this document, we present two simulation examples continued \\ from Section 4, additional numerical results of the real data applica- \\ tion from Section 5, and the proofs for Lemmas A.1-A.3.
}

S.1. Simulation studies. Example 2. In this example, we evaluate our proposed methods by conducting more simulation studies. The binary response $Y_{i}$ is generated from the logistic regression model given in (20) where $\alpha_{10}=0.5, \alpha_{11}\left(x_{1}\right)=4 \sin \left(2 \pi x_{1}\right), \alpha_{12}\left(x_{2}\right)=6 x_{2}-3, \alpha_{20}=0.5$, $\alpha_{21}\left(x_{1}\right)=4 \cos \left(2 \pi x_{1}\right), \alpha_{22}\left(x_{2}\right)=5\left\{\left(2 x_{2}-1\right)^{2}-1 / 3\right\}, \alpha_{30}=0.5, \alpha_{31}\left(x_{1}\right)=$ $6 x_{1}-3, \alpha_{32}\left(x_{2}\right)=4\left\{\sin \left(2 \pi x_{1}\right)+\cos \left(2 \pi x_{1}\right)\right\}$, and $\alpha_{\ell}\left(\mathbf{X}_{i}\right)=0$ for $l=4, \ldots, p$, so that 3 variables in $\mathbf{T}_{i}$ are important and the remaining $p-3$ variables in $\mathbf{T}_{i}$ are not important. We let $n=200$ and $p=50,200,500,1000$. The covariates $X_{i k}, k=1,2$, are simulated in the same way as in Example 1. The $T_{i \ell}$ are simulated genetic (SNP) effects, which have three possible genotype categories represented by AA, Aa and aa and coded as 2, 1, and 0 . In the context of model (4), the risk model we fit in this simulation is a multiplicative one. First we simulate $T_{i \ell}$ from an independent multinomial distribution with frequency $\left\{P_{A}^{2}, 2 P_{A}\left(1-P_{A}\right),\left(1-P_{A}\right)^{2}\right\}$ for (AA, Aa, aa).

\footnotetext{
* Ma's research was partially supported by NSF grant DMS-1306972

$\dagger$ Carroll's research was supported by a grant from the National Cancer Institute (U01CA057030).

¥ Corresponding author. Liang’s research was partially supported by NSF grants DMS1440121 and DMS-1418042 and by Award Number 11228103, made by National Natural Science Foundation of China.

AMS 2000 subject classifications: Primary 62G08; secondary 62G10, 62G20, 62J02 $62 \mathrm{~F} 12$

Keywords and phrases: Adaptive group LASSO, Bootstrap smoothing, Curse of dimensionality, Gene-environment interaction, Generalized additive partially linear models, Inference for high-dimensional data, Oracle property, Penalized likelihood, Polynomial splines, Two-step estimation, Undersmoothing
} 
We let $P_{A}=0.5$, which is the allele frequency for allele A. Next we simulate correlated $T_{i \ell}$ in the following way. We generate $Z_{i \ell}$ from the multivariate normal distribution with mean 0 , variance 1 and correlation coefficient 0.5 , and then we let $T_{i \ell}=2$ for $Z_{i \ell}<-0.5, T_{i \ell}=1$ for $-0.5 \leq Z_{i \ell} \leq 0.85$, and $T_{i \ell}=0$ for $Z_{i \ell}>0.85$. We use the empirical centered values of $T_{i \ell}$ to generate the binary response $Y_{i}$ from model (20) and fit the model. We conducted 500 replications for each simulation.

TABLE S.1

Variable selection and estimation results by the adaptive group lasso, the group lasso and logistic regression with lasso for $p=50,200,500,1000$ based on 500 replications. The columns of $C, O$ and $I$ show the percentage of correct-fitting, over-fitting, and incorrect-fitting. The columns TP, FP and MR show true positives, false positives and model errors, respectively.

\begin{tabular}{|c|cccccc|cccccccc|}
\hline & \multicolumn{1}{c|}{ Independent predictor } & \multicolumn{7}{c|}{ Correlated predictor } \\
\hline$p$ & C & O & I & TP & FP & MR & C & O & I & TP & FP & MR \\
\hline \hline \multicolumn{10}{|c|}{ Adaptive group lasso } \\
\hline 50 & 0.766 & 0.066 & 0.168 & 2.806 & 0.078 & 0.036 & 0.724 & 0.028 & 0.248 & 2.726 & 0.032 & 0.039 \\
\hline 200 & 0.658 & 0.140 & 0.202 & 2.776 & 0.200 & 0.043 & 0.642 & 0.074 & 0.284 & 2.686 & 0.094 & 0.046 \\
\hline 500 & 0.582 & 0.220 & 0.198 & 2.774 & 0.378 & 0.048 & 0.564 & 0.132 & 0.304 & 2.668 & 0.196 & 0.049 \\
\hline 1000 & 0.444 & 0.338 & 0.218 & 2.768 & 0.620 & 0.051 & 0.488 & 0.190 & 0.322 & 2.638 & 0.322 & 0.054 \\
\hline \hline \multicolumn{10}{|c|}{ Group lasso } \\
\hline 50 & 0.742 & 0.106 & 0.152 & 2.830 & 0.140 & 0.077 & 0.718 & 0.044 & 0.238 & 2.748 & 0.056 & 0.078 \\
\hline 200 & 0.564 & 0.260 & 0.176 & 2.814 & 0.488 & 0.085 & 0.650 & 0.120 & 0.230 & 2.748 & 0.172 & 0.084 \\
\hline 500 & 0.422 & 0.434 & 0.144 & 2.838 & 1.024 & 0.089 & 0.534 & 0.234 & 0.232 & 2.748 & 0.426 & 0.087 \\
\hline 1000 & 0.302 & 0.548 & 0.150 & 2.840 & 1.578 & 0.090 & 0.446 & 0.314 & 0.240 & 2.740 & 0.780 & 0.090 \\
\hline \hline \multicolumn{10}{|c}{} & Parametric logistic regression with adaptive group lasso \\
\hline 50 & 0.006 & 0.024 & 0.970 & 1.868 & 1.016 & 0.128 & 0.006 & 0.028 & 0.966 & 1.858 & 1.870 & 0.131 \\
\hline 200 & 0.002 & 0.014 & 0.984 & 1.830 & 3.196 & 0.127 & 0.000 & 0.010 & 0.990 & 1.808 & 5.934 & 0.118 \\
\hline 500 & 0.002 & 0.010 & 0.988 & 1.806 & 6.390 & 0.125 & 0.000 & 0.010 & 0.990 & 1.708 & 10.01 & 0.125 \\
\hline 1000 & 0.000 & 0.006 & 0.994 & 1.772 & 8.804 & 0.123 & 0.000 & 0.014 & 0.986 & 1.588 & 13.93 & 0.121 \\
\hline
\end{tabular}

The variable selection and estimation results by the penalized estimation procedures are summarized in Table S.1. The columns are the percentages of correct-fitting $(\mathrm{C})$, over-fitting $(\mathrm{O})$ and incorrect-fitting $(\mathrm{I})$, the average true positives (TP), the average false positives (FP) and the average model errors (MR). Table S.1 shows the results by using the adaptive group lasso (AGL), group lasso (GL) and by fitting a parametric logistic regression assuming linearity of the coefficient functions such that $\alpha_{\ell}\left(\mathbf{X}_{i}\right)=\alpha_{\ell 0}+\sum_{k=1}^{2} \beta_{\ell k} X_{i k}$ with the adaptive group lasso method.

Columns C, FP and MR show that, in all cases, the AGL method has larger correct fitting percentages and much smaller false positives and model errors than the GL method. The false positives and model errors using the GL are around twice as large as the corresponding values using the AGL. Column TP shows that the true positives with the AGL are slightly 
TABLE S.2

Computing time in seconds for generating one sample and estimation by the adaptive group Lasso method.

\begin{tabular}{|c|cccc|}
\hline$p$ & 50 & 200 & 500 & 1000 \\
\hline Independent predictor & 2.40 & 7.61 & 16.71 & 32.06 \\
\hline Correlated predictor & 1.95 & 6.48 & 15.67 & 34.72 \\
\hline
\end{tabular}

smaller than the true positives with GL. For the AGL method, the correct fitting percentages and the model errors are quite similar for independent and correlated predictors, which demonstrates that the proposed method for correlated predictors performs as well as for independent predictors. The true positives are quite similar for different $p$. By observing column $\mathrm{FP}$, for $p=1000$, the average number of false positives are 0.620 and 0.322 for the independent and correlated predictors, respectively, so that only 0.620 and 0.322 covariates are incorrectly selected among the 997 unimportant variables. Thus, the false positive rate by using the percentage of selected covariates among the unimportant covariates is close to 0 . For the parametric logistic regression results, we observe that the correct fitting percentages are almost 0 and the incorrect-fitting percentages are close to 1 for all cases. These results indicate that when we ignore the nonlinear interaction effects and treat the coefficients for the interactions as constants, some important covariates may not be identified for most replications. Table S.2 shows the computing time in seconds for generating one sample and estimation by the adaptive group Lasso method using iMac, $2.7 \mathrm{GHz}$ Intel Core: the computing time grows more slowly than $p / 25$ seconds.

To evaluate the performance of the two-step estimator for a given $k=1$ and $\ell=1,2,3$, we define the mean integrated squared error (MISE) as the average value of the integrated squared error $\operatorname{ISE}\left(\widehat{\alpha}_{\ell k}\right)=\sum_{i=1}^{n}\left\{\widehat{\alpha}_{\ell k}\left(X_{i k}\right)-\right.$ $\left.\alpha_{\ell k}\left(X_{i k}\right)\right\}^{2}$, where $\widehat{\alpha}_{\ell k}$ is an estimate of $\alpha_{\ell k}$. Table S.3 shows the MISE values for the two-step estimator $\widehat{\alpha}_{\ell k}^{\mathcal{S}}$, the one-step estimator $\widehat{\alpha}_{\ell k}$ and the infeasible oracle estimator $\widehat{\alpha}_{\ell k}^{\mathrm{OR}}$ that a priori knows $I_{1}$. We observe from Table S.3 that the one-step estimator $\widehat{\alpha}_{\ell k}$ has much larger MISE values than the other two estimators, and the two-step estimator has similar MISE values as the oracle estimator for all $p$ 's and for the two different covariate structures.

Next, we investigate the empirical coverage rates of the two SCBs given in (17) and (19). To calculate the unsmoothed and smoothed bootstrap standard deviations (16) and (18), we use $B=500$ bootstrap replications. The confidence bands are constructed at $L_{n}=20$ equally spaced points. At $95 \%$ confidence level, Table S.4 reports the empirical coverage rates (cov) and the sample averages of median and mean standard deviations (sd.median and 
4 SHUJIE MA, RAYMOND J. CARROLL, HUA LIANG, AND SHIZHONG XU

TABLE S.3

The MISE values for the two-step estimator $\widehat{\alpha}_{\ell k}^{\mathcal{S}}$, the one-step estimator $\widehat{\alpha}_{\ell k}$ and the oracle estimator $\widehat{\alpha}_{\ell k}^{O R}$ for $k=1$ and $\ell=1,2,3$ and for $p=50,200,500,1000$ based on 500 replications.

\begin{tabular}{|c|ccc|ccc|ccc|}
\hline \multicolumn{10}{|c|}{ Independent predictor } \\
\hline$p$ & $\widehat{\alpha}_{11}^{S}$ & $\widehat{\alpha}_{11}$ & $\widehat{\alpha}_{11}^{\mathrm{OR}}$ & $\widehat{\alpha}_{21}^{S}$ & $\widehat{\alpha}_{21}$ & $\widehat{\alpha}_{21}^{\mathrm{OR}}$ & $\widehat{\alpha}_{31}^{S}$ & $\widehat{\alpha}_{31}$ & $\widehat{\alpha}_{31}^{\mathrm{OR}}$ \\
\hline 50 & 1.116 & 2.324 & 0.954 & 1.127 & 2.215 & 1.170 & 1.076 & 1.879 & 0.965 \\
\hline 200 & 1.181 & 2.321 & 1.054 & 1.149 & 2.431 & 1.190 & 1.224 & 1.897 & 0.932 \\
\hline 500 & 1.255 & 2.519 & 1.000 & 1.182 & 2.483 & 1.152 & 1.222 & 1.890 & 0.986 \\
\hline 1000 & 1.201 & 2.513 & 0.972 & 1.239 & 2.649 & 1.236 & 1.249 & 2.008 & 1.154 \\
\hline
\end{tabular}

\begin{tabular}{|c|ccc|ccc|ccc|}
\hline \multicolumn{10}{|c|}{ Correlated predictor } \\
\hline$p$ & $\widehat{\alpha}_{11}^{S}$ & $\widehat{\alpha}_{11}$ & $\widehat{\alpha}_{11}^{\text {OR }}$ & $\widehat{\alpha}_{12}^{S}$ & $\widehat{\alpha}_{12}$ & $\widehat{\alpha}_{12}^{\text {OR }}$ & $\widehat{\alpha}_{13}^{S}$ & $\widehat{\alpha}_{13}$ & $\widehat{\alpha}_{13}^{\text {OR }}$ \\
\hline 50 & 1.196 & 2.869 & 1.147 & 1.348 & 2.689 & 1.455 & 1.236 & 1.964 & 1.101 \\
\hline 200 & 1.306 & 2.997 & 1.178 & 1.368 & 2.816 & 1.503 & 1.356 & 2.036 & 1.151 \\
\hline 500 & 1.325 & 3.056 & 1.158 & 1.477 & 2.888 & 1.540 & 1.347 & 2.053 & 1.111 \\
\hline 1000 & 1.389 & 3.259 & 1.153 & 1.418 & 2.983 & 1.449 & 1.420 & 2.151 & 1.119 \\
\hline
\end{tabular}

TABLE S.4

The empirical coverage rates (cov) and the sample average of median and mean of the standard deviations (sd.median and sd.mean) for the unsmoothed $S C B$ (17) and smoothed $S C B$ (19) for the coefficient functions $\alpha_{11}\left(x_{1}\right)$ and $\alpha_{21}\left(x_{1}\right)$ at $n=200$. IND and COR denote that the components in $\mathbf{T}_{i}$ are generated independently and with correlations, respectively.

\begin{tabular}{|c|c|ccc|ccc|}
\hline \multicolumn{2}{|c|}{} & \multicolumn{3}{|c|}{ unsmoothed bootstrap } & \multicolumn{3}{c|}{ smoothed bootstrap } \\
\cline { 3 - 8 } \multicolumn{2}{|c|}{} & cov & sd.median & sd.mean & cov & sd.median & sd.mean \\
\hline IND & $\alpha_{11}$ & 0.856 & 1.074 & 1.274 & 0.934 & 1.064 & 1.248 \\
& $\alpha_{21}$ & 0.880 & 1.074 & 1.346 & 0.950 & 1.054 & 1.304 \\
\hline COR & $\alpha_{11}$ & 0.854 & 1.099 & 1.324 & 0.930 & 1.092 & 1.310 \\
& $\alpha_{21}$ & 0.866 & 1.110 & 1.398 & 0.946 & 1.104 & 1.378 \\
\hline
\end{tabular}


sd.mean), respectively for the unsmoothed SCB (17) and smoothed SCB (19) for coefficient functions $\alpha_{11}\left(x_{1}\right)$ and $\alpha_{21}\left(x_{1}\right)$. We see that the smoothed bootstrap method has empirical coverage rates closer to the nominal confidence level 0.95 with even smaller standard deviations comparing to the traditional resampling method.

Example 3. In this example, we simulate data from a parametric logistic regression model and compare the variable selection and estimation performance of different methods. Same as Example 1 of Section 4, we use 1286 SNPs located on the sixth chromosome from the Framingham Heart Study to simulate the binary response from the logistic model

$$
\begin{aligned}
& \operatorname{logit}\left\{P\left(Y_{i}=1 \mid \mathbf{X}_{i}, \mathbf{T}_{i}\right)\right\} \\
& =\alpha_{0}+\sum_{k=1}^{2} \alpha_{0 k} X_{i k}+\sum_{\ell=1}^{p} \alpha_{\ell} T_{i \ell}+\sum_{\ell=1}^{p} \sum_{k=1}^{2} \alpha_{\ell k} X_{i k} T_{i \ell} \\
& =\sum_{\ell=0}^{p}\left(\alpha_{\ell}+\sum_{k=1}^{2} \alpha_{\ell k} X_{i k}\right) T_{i \ell},
\end{aligned}
$$

where $T_{i 0}=1$, for $\ell=1, \ldots, 1286$, with the four SNPs as described in Example 1 as important covariates and the other SNPs as unimportant covariates, so that $s=4$ (the number of important covariates), $p=1286$, and the sample size $n=300$. Clearly, the above logistic model is a special case of the GACM (20) by assuming that the coefficient functions have a linear structure. The covariates $X_{i k}, k=1,2$, are simulated environmental effects, which are generated from independent uniform distributions on $[0,1]$. We let $\beta_{\ell}=3$ and $\beta_{\ell k}=3$ for $\ell=0, \ldots, 4$ and $\beta_{\ell}=0$ and $\beta_{\ell k}=0$ for $\ell=5, \ldots, 1286$. We conducted 500 replications for each simulation. We fit the data with the proposed GACM (20), the GVCM ((Lian, 2012)) and the parametric logistic model by using the adaptive group lasso (AGL) and group lasso (GL).

The variable selection and estimation results are summarized in Table S.5. The columns are the percentages of correct-fitting (C), over-fitting (O) and incorrect-fitting (I), the average true positives (TP), the average false positives (FP) and the average model errors (MR), which are defined in Example 1 of Section 4. The parametric logistic model has a better variable selection result than the other two models with larger true positives and smaller false positives observed. This result is not surprising, since the logistic model is the true model from which the data are generated. The GACM has a comparable variable selection result to the logistic model by observing similar values of true positives and incorrect-fitting percentages. Moreover, by the AGL method, the GACM has a smaller model error than the logistic model, since the GACM may provide a more robust estimator. The GVCM 
has worse vriable selection and estimation results than the other two models for all cases.

TABLE S.5

Results of Example 3 in the simulation studies. Variable selection and estimation results by the adaptive group lasso and the group lasso with the GACM, GVCM and parametric logistic regression, respectively, based on 500 replications. The columns of $C, O$ and $I$ show the percentage of correct-fitting, over-fitting, and incorrect-fitting. The columns TP, FP and MR show true positives, false positives and model errors, respectively.

\begin{tabular}{|c|c|cccccc|}
\hline & & C & O & I & TP & FP & MR \\
\hline GACM & AGL & 0.690 & 0.270 & 0.040 & 3.956 & 0.405 & 0.075 \\
& GL & 0.540 & 0.430 & 0.030 & 3.970 & 0.705 & 0.103 \\
\hline GVCM & AGL & 0.604 & 0.290 & 0.106 & 3.894 & 0.410 & 0.095 \\
& GL & 0.490 & 0.410 & 0.100 & 3.900 & 0.700 & 0.109 \\
\hline Logistic regression & AGL & 0.830 & 0.144 & 0.026 & 3.974 & 0.176 & 0.087 \\
& GL & 0.554 & 0.430 & 0.016 & 3.984 & 0.576 & 0.090 \\
\hline
\end{tabular}

S.2. Data application. Figure S.1 depicts the plots of $\widehat{m}_{k}(\cdot)$ for $k=$ $1,2,3$ by one-step cubic spline estimation by fitting the model 21 given in Section 5 for nonlinear main effects of the environmental factors. See Wang et al. (2011) for the estimation procedure. Clearly the estimate of each nonparametric function has a nonlinear pattern. For example, Figure S.1 (a) shows that the chance of developing obesity decreases as the sleeping hours increase when people have no less than 4 hours sleep per day. This result corroborates findings by scientists (Gangwisch et al., 2005) that there is a correlation between a steep rise in obesity and less sleep. However, for people with less than 4 hours sleep per day, the value of $\widehat{m}_{k}(\cdot)$ increases with increasing sleeping hours. It has been shown that too little sleep is also linked to some other serious health risks such as colon cancer (Thompson et al., 2011), and we speculate that this may be one cause of weight loss. From Figure S.1 (b), we observe a nonlinear decreasing pattern of the estimated function with activity hours with the curve becoming steeper as activity hours increase, which indicates that physical activity can lower the risk of developing obesity, a reasonably intuitive result. Figure S.1 (c) shows a reverse change pattern of the estimated curve as Figure S.1 (b). We clearly see that diastolic blood pressure has a positive correlation with obesity. These plots give us a visual impression that the conditional mean of the response changes nonlinearly with those environmental factors.

\section{S.3. Proof of Lemma A.1.}


(a)

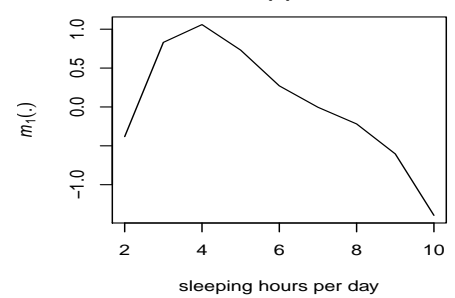

(c)

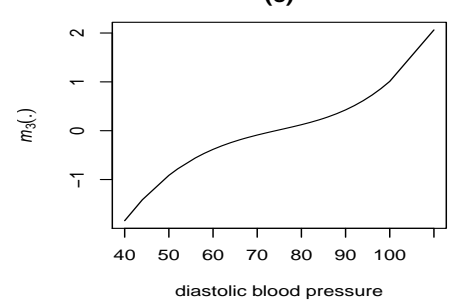

(b)

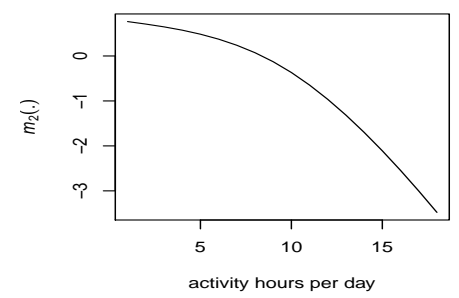

FIG S.1. Plots of the spline estimators $\widehat{m}_{k}(\cdot)$ of the nonparametric functions $m_{k}(\cdot)$ for $k=1,2,3$ based on model (21).

S.1. Proof of (25). In the following, we will provide the proofs for the result (25). Let $k_{n}=\lambda_{n}\left\|w_{n, I_{1}}\right\|+n^{-1 / 2} J_{n}^{1 / 2}+J_{n}^{-r}$. We will show that for any given $\epsilon>0$, there exists a large constant $C>0$ such that

$$
P\left\{\inf _{\|\mathbf{u}\|_{2}=C} L_{n}\left(\gamma_{I_{1}}+k_{n} \mathbf{u}\right)>L_{n}\left(\gamma_{I_{1}}\right)\right\} \geq 1-\epsilon .
$$

Let $S=\left\{\gamma_{I_{1}}+k_{n} \mathbf{u}:\|\mathbf{u}\|_{2} \leq C\right\}$ be the ball defined in a neighborhood of $\gamma_{I_{1}}$. By the above condition, it implies that with probability at least $1-\epsilon$, there exists a local minimum for (24) in the interior of $S$. By convexity of $L_{n}\left(\gamma_{I_{1}}\right)$ as assumed in Condition (C2), this local minimizer is the global minimizer $\widehat{\gamma}_{I_{1}}^{0}$ of $(24)$, and hence we have $\left\|\widehat{\gamma}_{I_{1}}^{0}-\gamma_{I_{1}}\right\|_{2}=O_{p}\left(k_{n}\right)$. Let $\widetilde{\gamma}_{I_{1}}=$ $\gamma_{I_{1}}+k_{n} \mathbf{u}=\left(\widetilde{\gamma}_{\ell}: \ell \in I_{1}\right)^{\mathrm{T}}$ with $\|\mathbf{u}\|_{2}=C$, and $D_{n}(\mathbf{u})=L_{n}\left(\widetilde{\gamma}_{I_{1}}\right)-L_{n}\left(\gamma_{I_{1}}\right)=$ $D_{n 1}(\mathbf{u})+D_{n 2}(\mathbf{u})$, where

$$
\begin{aligned}
D_{n 1}(\mathbf{u})= & \sum_{i=1}^{n} Q\left\{g^{-1}\left(\sum_{\ell \in I_{1}} B\left(\mathbf{X}_{i}\right)^{\mathrm{T}} \widetilde{\boldsymbol{\gamma}}_{\ell} T_{i \ell}\right), Y_{i}\right\} \\
& -\sum_{i=1}^{n} Q\left\{g^{-1}\left(\sum_{\ell \in I_{1}} B\left(\mathbf{X}_{i}\right)^{\mathrm{T}} \boldsymbol{\gamma}_{\ell} T_{i \ell}\right), Y_{i}\right\}, \\
D_{n 2}(\mathbf{u})= & n \lambda_{n} \sum_{\ell \in I_{1}} w_{n \ell}\left\|\widetilde{\boldsymbol{\gamma}}_{\ell}\right\|_{2}-n \lambda_{n} \sum_{\ell \in I_{1}} w_{n \ell}\left\|\boldsymbol{\gamma}_{\ell}\right\|_{2} .
\end{aligned}
$$

To prove (S.1), it is equivalent to show that with probability approaching 1 , for large enough $C, D_{n}(\mathbf{u})>0$. By Taylor expansion, $D_{n 1}(\mathbf{u})=D_{n 1,1}(\mathbf{u})+$ 
$D_{n 1,2}(\mathbf{u})$, where

$$
\begin{aligned}
& D_{n 1,1}(\mathbf{u})=k_{n} \sum_{i=1}^{n} q_{1}\left\{\sum_{\ell \in I_{1}} \alpha_{\ell}^{0}\left(\mathbf{X}_{i}\right) T_{i \ell}, Y_{i}\right\}\left\{\sum_{\ell \in I_{1}} B\left(\mathbf{X}_{i}\right)^{\mathrm{T}} \mathbf{u} T_{i \ell}\right\}, \\
& D_{n 1,2}(\mathbf{u})=k_{n}^{2} \sum_{i=1}^{n} q_{2}\left\{\sum_{\ell \in I_{1}} \alpha_{\ell}^{*}\left(\mathbf{X}_{i}\right) T_{i \ell}, Y_{i}\right\}\left\{\sum_{\ell \in I_{1}} B\left(\mathbf{X}_{i}\right)^{\mathrm{T}} \mathbf{u} T_{i \ell}\right\}^{2},
\end{aligned}
$$

where $\alpha_{\ell}^{*}\left(\mathbf{X}_{i}\right)=B\left(\mathbf{X}_{i}\right)^{\mathrm{T}} \boldsymbol{\gamma}_{\ell}^{*}$ with $\boldsymbol{\gamma}_{\ell}^{*} \in\left(\boldsymbol{\gamma}_{\ell}, \widetilde{\gamma}_{\ell}\right)$. We next will find lower bounds for $D_{n 1,1}(\mathbf{u}), D_{n 1,2}(\mathbf{u})$ and $D_{n 2}(\mathbf{u})$, respectively, through the following three steps.

Step I. Let

$$
\varrho_{i}=g^{-1}\left\{\eta\left(\mathbf{X}_{i}, \mathbf{T}_{i}\right)\right\}-g^{-1}\left\{\eta_{I_{1}}^{0}\left(\mathbf{X}_{i}, \mathbf{T}_{i}\right)\right\}, \eta_{I_{1}}^{0}\left(\mathbf{X}_{i}, \mathbf{T}_{i}\right)=\sum_{\ell \in I_{1}} \alpha_{\ell}^{0}\left(\mathbf{X}_{i}\right) T_{i \ell}
$$

Then $\sum_{i=1}^{n} q_{1}\left\{\sum_{\ell \in I_{1}} \alpha_{\ell}^{0}\left(\mathbf{X}_{i}\right) T_{i \ell}, Y_{i}\right\}\left\{\sum_{\ell \in I_{1}} B\left(\mathbf{X}_{i}\right) T_{i \ell}\right\}=-\sum_{\ell \in I_{1}}\left(\xi_{1 \ell}+\xi_{2 \ell}\right)$, where

$$
\begin{aligned}
& \xi_{1 \ell}=\sum_{i=1}^{n} \varepsilon_{i} \rho_{1}\left\{\eta_{I_{1}}^{0}\left(\mathbf{X}_{i}, \mathbf{T}_{i}\right)\right\} B\left(\mathbf{X}_{i}\right) T_{i \ell} \\
& \xi_{2 \ell}=\sum_{i=1}^{n} \varrho_{i} \rho_{1}\left\{\eta_{I_{1}}^{0}\left(\mathbf{X}_{i}, \mathbf{T}_{i}\right)\right\} B\left(\mathbf{X}_{i}\right) T_{i \ell}
\end{aligned}
$$

Then with probability approaching 1 , by Conditions (C4) and (C5),

$$
\left\|\xi_{1 \ell}\right\|_{2}^{2} \asymp \sum_{j, k} \sum_{i=1}^{n} \varepsilon_{i}^{2}\left\{\rho_{1}\left\{\eta_{I_{1}}^{0}\left(\mathbf{X}_{i}, \mathbf{T}_{i}\right)\right\} B_{j, k}\left(X_{i k}\right) T_{i \ell}\right\}^{2} \asymp n J_{n}
$$

and

$$
\left\|\xi_{2 \ell}\right\|_{2}^{2} \asymp J_{n} n^{2}\left[E\left\{\rho_{1}\left(\eta_{I_{1}}^{0}\left(\mathbf{X}_{i}, \mathbf{T}_{i}\right)\right) B_{j, k}\left(X_{i k}\right) T_{i \ell}\right\}\right]^{2}=O\left(n^{2} J_{n}^{-2 r}\right) .
$$

Thus, $\left\|-\sum_{\ell \in I_{1}}\left(\xi_{1 \ell}+\xi_{2 \ell}\right)\right\|_{2} \leq\left\{2 s\left(\left\|\xi_{1 \ell}\right\|_{2}^{2}+\left\|\xi_{2 \ell}\right\|_{2}^{2}\right)\right\}^{1 / 2}=O_{p}\left(n^{1 / 2} J_{n}^{1 / 2}+\right.$ $\left.n J_{n}^{-r}\right)$. Therefore with probability approaching 1 , there exists a constant $1<c_{1}<\infty$, such that

$$
\left|D_{n 1,1}(\mathbf{u})\right| \leq C k_{n}\left\|-\sum_{\ell \in I_{1}}\left(\xi_{1 \ell}+\xi_{2 \ell}\right)\right\|_{2} \leq C c_{1} n k_{n}\left(n^{-1 / 2} J_{n}^{1 / 2}+J_{n}^{-r}\right) .
$$

Step II. By Theorem 5.4.2 of DeVore and Lorentz (1993), for sufficiently large $n$, it can be proved that there exist constants $0<c_{B} \leq C_{B}<\infty$, for any $\mathbf{a}=\left(a_{j k}: 1 \leq j \leq J_{n}, 1 \leq k \leq d\right)$, such that

$$
c_{B} \mathbf{a}^{\mathrm{T}} \mathbf{a} \leq \mathbf{a}^{\mathrm{T}} E\left(B B^{\mathrm{T}}\right) \mathbf{a} \leq C_{B} \mathbf{a}^{\mathrm{T}} \mathbf{a} .
$$


and by Bernstein's inequality (Bosq, 1998), we can prove

$$
\begin{aligned}
& \sup _{1 \leq j, j^{\prime} \leq J_{n}, 1 \leq k \leq d} \mid n^{-1} \sum_{i=1}^{n}\left\{B_{j, k}\left(X_{i k}\right) B_{j^{\prime}, k}\left(X_{i k}\right)\right. \\
&\left.-E\left(B_{j, k}\left(X_{i k}\right) B_{j^{\prime}, k}\left(X_{i k}\right)\right)\right\} \mid=O_{a . s .}\left(\sqrt{J_{n} n^{-1} \log n}\right), \\
& \sup _{1 \leq j, j^{\prime} \leq J_{n}, 1 \leq k \neq k^{\prime} \leq d} \mid n^{-1} \sum_{i=1}^{n}\left\{B_{j, k}\left(X_{i k}\right) B_{j^{\prime}, k^{\prime}}\left(X_{i k^{\prime}}\right)\right. \\
&\left.-E\left(B_{j, k}\left(X_{i k}\right) B_{j^{\prime}, k^{\prime}}\left(X_{i k^{\prime}}\right)\right)\right\} \mid=O_{a . s .}\left(\sqrt{n^{-1} \log n}\right) .
\end{aligned}
$$

By the above results, we have for $J_{n} \sqrt{n^{-1} \log n}=o(1)$, with probability 1 , as $n \rightarrow \infty$,

$$
c_{B} \mathbf{a}^{\mathrm{T}} \mathbf{a} \leq \mathbf{a}^{\mathrm{T}} n^{-1} \sum_{i=1}^{n}\left\{B\left(\mathbf{X}_{i}\right) B\left(\mathbf{X}_{i}\right)^{\mathrm{T}}\right\} \mathbf{a} \leq C_{B} \mathbf{a}^{\mathrm{T}} \mathbf{a}
$$

Thus,

$D_{n 1,2}(\mathbf{u}) \asymp k_{n}^{2} \sum_{i=1}^{n} \rho_{2}\left\{\sum_{\ell \in I_{1}} \alpha_{\ell}^{*}\left(\mathbf{X}_{i}\right) T_{i \ell}\right\} \mathbf{u}^{\mathrm{T}} B\left(\mathbf{X}_{i}\right) B\left(\mathbf{X}_{i}\right)^{\mathrm{T}} \mathbf{u} \geq k_{n}^{2} n c_{\rho_{2}} c_{B} C^{2}$,

for a constant $0<c_{\rho_{2}}<\infty$.

Step III. It is easy to see that there exists a constant $0<c_{2}<\infty$, such that

$\left|D_{n 2}(\mathbf{u})\right|=\left|n \lambda_{n} \sum_{\ell \in I_{1}} w_{n \ell}\left\|\widetilde{\gamma}_{\ell}\right\|_{2}-n \lambda_{n} \sum_{\ell \in I_{1}} w_{n \ell}\left\|\gamma_{\ell}\right\|_{2}\right| \leq C c_{2} n \lambda_{n} k_{n}\left\|w_{n, I_{1}}\right\|$.

Thus by (S.4), (S.6), and (S.7) in Steps I, II and III and the fact that $k_{n}=\lambda_{n}\left\|w_{n, I_{1}}\right\|+n^{-1 / 2} J_{n}^{1 / 2}+J_{n}^{-r}$, one has with probability approaching 1 , for large enough $C$,

$$
\begin{aligned}
D_{n}(\mathbf{u}) & =D_{n 1,1}(\mathbf{u})+D_{n 1,2}(\mathbf{u})+D_{n 2}(\mathbf{u}) \\
& \geq k_{n}^{2} n c_{\rho_{2}} c_{B} C^{2}-C c_{1} n k_{n}\left(n^{-1 / 2} J_{n}^{1 / 2}+J_{n}^{-r}\right)-C c_{2} n k_{n} \lambda_{n}\left\|w_{n, I_{1}}\right\| \\
& \geq k_{n}^{2} n c_{\rho_{2}} c_{B} C^{2}-C\left(c_{1} \vee c_{2}\right) n k_{n}^{2}>0 .
\end{aligned}
$$

Therefore, Condition (S.1) is satisfied, and hence we have

$$
\left\|\widehat{\gamma}_{I_{1}}^{0}-\gamma_{I_{1}}\right\|_{2}=O_{p}\left(\lambda_{n}\left\|w_{n, I_{1}}\right\|+n^{-1 / 2} J_{n}^{1 / 2}+J_{n}^{-r}\right) .
$$

This proves the result $(25)$ 
S.2. Proof of (26). In the following, we will provide the proofs for the result (26). By the definition of $\widehat{\gamma}_{I_{1}}^{0}=\left(\widehat{\gamma}_{\ell}^{0 \mathrm{~T}}: \ell \in I_{1}\right)^{\mathrm{T}}$, (S.9)

$\sum_{i=1}^{n} q_{1}\left\{\sum_{\ell \in I_{1}} B\left(\mathbf{X}_{i}\right)^{\mathrm{T}} \widehat{\gamma}_{\ell}^{0} T_{i \ell}, Y_{i}\right\} B\left(\mathbf{X}_{i}\right) T_{i \ell}+n \lambda_{n} w_{n \ell} \widehat{\gamma}_{\ell}^{0} /\left\|\widehat{\gamma}_{\ell}^{0}\right\|_{2}=0, \ell \in I_{1}$.

A necessary and sufficient condition for $P\left\{\widehat{\gamma}=\left(\widehat{\gamma}_{I_{1}}^{0 \mathrm{~T}}, \widehat{\gamma}_{I_{2}}^{0 \mathrm{~T}}\right)^{\mathrm{T}}\right\} \rightarrow 1$ is

$$
\sum_{i=1}^{n} q_{1}\left(\sum_{\ell \in I_{1}} B\left(\mathbf{X}_{i}\right)^{\mathrm{T}} \widehat{\gamma}_{\ell}^{0} T_{i \ell}+\sum_{\ell \in I_{2}} B\left(\mathbf{X}_{i}\right)^{\mathrm{T}} \widehat{\gamma}_{\ell}^{0} T_{i \ell}, Y_{i}\right) B\left(\mathbf{X}_{i}\right) T_{i \ell}
$$

$$
\left\|\sum_{i=1}^{n} q_{1}\left\{\sum_{\ell \in I_{1}} B\left(\mathbf{X}_{i}\right)^{\mathrm{T}} \widehat{\gamma}_{\ell}^{0} T_{i \ell}+\sum_{\ell \in I_{2}} B\left(\mathbf{X}_{i}\right)^{\mathrm{T}} \widehat{\gamma}_{\ell}^{0} T_{i \ell}, Y_{i}\right\} B\left(\mathbf{X}_{i}\right) T_{i \ell}\right\|_{2}
$$

$$
\leq n \lambda_{n} w_{n \ell}, \ell \in I_{2}
$$

with probability approaching 1. By the Karush-Kuhn-Tucker (KKT) conditions as given in the literature such as equation (31) in Huang, Horowitz and Wei (2010) and equations (7) and (8) in Tibshirani et al. (2010), the estimator satisfying the conditions in (S.10) and (S.11) is the minimizer of the objective function $L_{n}(\boldsymbol{\gamma})$ in (8). Thus we have $P\left\{\widehat{\gamma}=\left(\widehat{\gamma}_{I_{1}}^{0 \mathrm{~T}}, \widehat{\boldsymbol{\gamma}}_{I_{2}}^{0 \mathrm{~T}}\right)^{\mathrm{T}}\right\} \rightarrow 1$. Equation (S.10) is implied directly by (S.9), since $\sum_{\ell \in I_{2}} B\left(\mathbf{X}_{i}\right)^{\mathrm{T}} \widehat{\boldsymbol{\gamma}}_{\ell}^{0} T_{i \ell}=0$ by the definition of $\widehat{\gamma}_{I_{2}}^{0}$. In the following, we will prove (S.11). By Taylor expansion,

$$
\begin{gathered}
\max _{\ell \in I_{2}}\left\|\sum_{i=1}^{n} q_{1}\left\{\sum_{\ell \in I_{1}} B\left(\mathbf{X}_{i}\right)^{\mathrm{T}} \widehat{\gamma}_{\ell}^{0} T_{i \ell}+\sum_{\ell \in I_{2}} B\left(\mathbf{X}_{i}\right)^{\mathrm{T}} \widehat{\boldsymbol{\gamma}}_{\ell}^{0} T_{i \ell}, Y_{i}\right\} B\left(\mathbf{X}_{i}\right) T_{i \ell}\right\|_{2} \\
\leq \Gamma_{1 n}+\Gamma_{2 n}
\end{gathered}
$$

where

$$
\begin{aligned}
\Gamma_{1 n}= & \max _{\ell \in I_{2}}\left\|\sum_{i=1}^{n} q_{1}\left(\sum_{\ell \in I_{1}} \alpha_{\ell}^{0}\left(\mathbf{X}_{i}\right) T_{i \ell}, Y_{i}\right) B\left(\mathbf{X}_{i}\right) T_{i \ell}\right\|_{2}, \\
\Gamma_{2 n}= & \max _{\ell \in I_{2}} \| \sum_{i=1}^{n} q_{2}\left(\sum_{\ell \in I_{1}} \alpha_{\ell}^{*}\left(\mathbf{X}_{i}\right) T_{i \ell}, Y_{i}\right) \times \\
& \left\{\sum_{\ell \in I_{1}} B\left(\mathbf{X}_{i}\right)^{\mathrm{T}}\left(\widehat{\gamma}_{\ell}^{0}-\gamma_{\ell}\right) T_{i \ell}\right\} B\left(\mathbf{X}_{i}\right) T_{i \ell} \|_{2},
\end{aligned}
$$

in which $\alpha_{\ell}^{*}\left(\mathbf{X}_{i}\right)=B_{i}^{\mathrm{T}}\left(\mathbf{X}_{i}\right) \boldsymbol{\gamma}_{\ell}^{*}$, with $\boldsymbol{\gamma}_{\ell}^{*} \in\left(\boldsymbol{\gamma}_{\ell}, \widehat{\gamma}_{\ell}^{0}\right)$.

$$
\begin{aligned}
\Gamma_{1 n} \leq & \left(d J_{n}+1\right)^{1 / 2} \max _{\ell \in I_{2}}\left\|\sum_{i=1}^{n} \varepsilon_{i} \rho_{1}\left\{\eta_{I_{1}}^{0}\left(\mathbf{X}_{i}, \mathbf{T}_{i}\right)\right\} B\left(\mathbf{X}_{i}\right) T_{i \ell}\right\|_{\infty} \\
& +\left(d J_{n}+1\right)^{1 / 2} \max _{\ell \in I_{2}}\left\|\sum_{i=1}^{n} \varrho_{i} \rho_{1}\left\{\eta_{I_{1}}^{0}\left(\mathbf{X}_{i}, \mathbf{T}_{i}\right)\right\} B\left(\mathbf{X}_{i}\right) T_{i \ell}\right\|_{\infty},
\end{aligned}
$$

where $\varrho_{i}$ is defined in (S.2). By Conditions (C4) and (C5), it can be proved by Bernstein's inequality (Bosq, 1998) that

$$
\max _{\ell \in I_{2}}\left\|\sum_{i=1}^{n} \varepsilon_{i} \rho_{1}\left\{\eta_{I_{1}}^{0}\left(\mathbf{X}_{i}, \mathbf{T}_{i}\right)\right\} B\left(\mathbf{X}_{i}\right) T_{i \ell}\right\|_{\infty}=O_{p}\left(n^{1 / 2} \sqrt{\log \left(p J_{n}\right)}\right) .
$$


Following the same reasoning as (S.3), one has

$$
\max _{\ell \in I_{2}}\left\|\sum_{i=1}^{n} \varrho_{i} \rho_{1}\left\{\eta_{I_{1}}^{0}\left(\mathbf{X}_{i}, \mathbf{T}_{i}\right)\right\} B\left(\mathbf{X}_{i}\right) T_{i \ell}\right\|_{\infty}=O_{p}\left(n J_{n}^{-1 / 2} J_{n}^{-r}\right) .
$$

Thus $\Gamma_{1 n}=O_{p}\left(n^{1 / 2} J_{n}^{1 / 2} \sqrt{\log \left(p J_{n}\right)}+n J_{n}^{-r}\right)$. By (S.8),

$$
\begin{aligned}
\Gamma_{2 n} & \asymp \max _{\ell \in I_{2}}\left\|\sum_{i=1}^{n} B\left(\mathbf{X}_{i}\right) T_{i \ell}\right\|_{2} O_{p}\left(\lambda_{n}\left\|w_{n, I_{1}}\right\|+n^{-1 / 2} J_{n}^{1 / 2}+J_{n}^{-r}\right) \\
& =O_{p}\left(n \lambda_{n}\left\|w_{n, I_{1}}\right\|+n^{1 / 2} J_{n}^{1 / 2}+n J_{n}^{-r}\right) .
\end{aligned}
$$

Thus

$$
\begin{aligned}
\max _{\ell \in I_{2}} \| & \sum_{i=1}^{n} q_{1}\left(\sum_{\ell \in I_{1}} B\left(\mathbf{X}_{i}\right)^{\mathrm{T}} \widehat{\gamma}_{\ell} T_{i \ell}+\sum_{\ell \in I_{2}} B\left(\mathbf{X}_{i}\right)^{\mathrm{T}} \widehat{\gamma}_{\ell} T_{i \ell}, Y_{i}\right) B\left(\mathbf{X}_{i}\right) T_{i \ell} \|_{2} \\
= & O_{p}\left(n \lambda_{n}\left\|w_{n, I_{1}}\right\|+n^{1 / 2} J_{n}^{1 / 2} \sqrt{\log \left(p J_{n}\right)}+n J_{n}^{-r}\right) .
\end{aligned}
$$

Therefore, under the assumptions in Theorem 1, (S.11) holds. Thus, the result (26) is proved.

S.4. Proof of Lemma A.3. We first prove the result (S.12) given below, which will be used in the proof of the result (27).

$$
\left\|\partial L_{n}^{\mathcal{S}}\left(\widehat{\gamma}_{, 1}^{\mathrm{OR}}\right) / \partial \widehat{\gamma}_{, 1}^{\mathrm{OR}}\right\|_{\infty}=O_{p}\left(\sqrt{n \log n / J_{n}^{\mathcal{S}}}+n\left(J_{n}^{\mathcal{S}}\right)^{-1 / 2}\left(J_{n}^{\mathrm{ini}}\right)^{-r}\right) .
$$

Under Assumptions 1-3, by Theorem 1, one has with probability approaching $1, \widehat{I}_{1}=I_{1}$. It can be proved that $\left\|\widehat{\gamma}_{\widehat{I}_{1}}^{\text {ini }}-\gamma_{\widehat{I}_{1}}\right\|_{2}=O_{p}\left(n^{-1 / 2} J_{n}^{1 / 2}+\left(J_{n}^{\text {ini }}\right)^{-r}\right)$. For any vectors $\boldsymbol{\gamma}_{, 1}^{\mathcal{S}}=\left(\left(\boldsymbol{\gamma}_{\ell 1}^{\mathcal{S}}\right)^{\mathrm{T}}, \ell \in \widehat{I}_{1}\right)^{\mathrm{T}}$ and $\boldsymbol{\gamma}_{-1}=\left(\boldsymbol{\gamma}_{\ell k}^{\mathrm{T}}, k \geq 2, \ell \in \widehat{I}_{1}\right)^{\mathrm{T}}$, denote

$$
\begin{aligned}
L_{n}^{*}\left(\boldsymbol{\gamma}_{, 1}^{\mathcal{S}}, \boldsymbol{\gamma}_{-1}\right)= & \sum_{i=1}^{n} Q\left[g ^ { - 1 } \left\{\sum_{\ell \in \widehat{I}_{1}} B_{1}^{\mathcal{S}}\left(X_{i 1}\right)^{\mathrm{T}} \boldsymbol{\gamma}_{\ell 1}^{\mathcal{S}} T_{i \ell}\right.\right. \\
& \left.\left.+\sum_{\ell \in \widehat{I}_{1}} \sum_{k \geq 2} B_{k}\left(X_{i k}\right)^{\mathrm{T}} \boldsymbol{\gamma}_{\ell k} T_{i \ell}\right\}, Y_{i}\right] .
\end{aligned}
$$

Let $\widehat{\gamma}_{,-1}^{\text {ini }}=\left(\left(\widehat{\gamma}_{\ell k}^{\text {ini }}\right)^{\mathrm{T}}, k \geq 2, \ell \in \widehat{I}_{1}\right)^{\mathrm{T}}$. We will show that

$$
\begin{aligned}
& \left\|\partial L_{n}^{*}\left(\widehat{\gamma}_{, 1}^{\mathrm{OR}}, \widehat{\gamma}_{,-1}^{\mathrm{ini}}\right) / \partial \widehat{\gamma}_{, 1}^{\mathrm{OR}}-\partial L_{n}^{*}\left(\widehat{\gamma}_{, 1}^{\mathrm{OR}}, \gamma_{-1}\right) / \partial \widehat{\gamma}_{, 1}^{\mathrm{OR}}\right\|_{\infty} \\
= & O_{p}\left(\sqrt{n \log n / J_{n}^{\S}}+n\left(J_{n}^{\mathcal{S}}\right)^{-1 / 2}\left(J_{n}^{\mathrm{ini}}\right)^{-r}\right) .
\end{aligned}
$$

By Theorem 1, with probability approaching 1, we have

$$
\begin{aligned}
L_{n}^{\mathrm{OR}}\left(\widehat{\gamma}_{, 1}^{\mathrm{OR}}\right) & =\sum_{i=1}^{n} Q\left[g^{-1}\left\{\sum_{\ell \in \widehat{I}_{1}} B_{1}^{\mathcal{S}}\left(X_{i 1}\right)^{\mathrm{T}} \widehat{\boldsymbol{\gamma}}_{\ell 1}^{\mathrm{OR}} T_{i \ell}+\sum_{\ell \in \widehat{I}_{1}} \alpha_{\ell,-1}\left(\mathbf{X}_{i}\right) T_{i \ell}\right\}, Y_{i}\right] \\
(\mathrm{S} .15) & =L_{n}^{*}\left(\widehat{\gamma}_{, 1}^{\mathrm{OR}}, \gamma_{-1}\right)+O\left(n\left(J_{n}^{\mathcal{S}}\right)^{-1 / 2}\left(J_{n}^{\mathrm{ini}}\right)^{-r}\right) .
\end{aligned}
$$


By definitions in (11) and (S.13), we have

$$
\partial L_{n}^{*}\left(\widehat{\gamma}_{, 1}^{\mathrm{OR}}, \widehat{\gamma}_{,-1}^{\mathrm{ini}}\right) / \partial \widehat{\gamma}_{, 1}^{\mathrm{OR}}=\partial L_{n}^{\mathcal{S}}\left(\widehat{\gamma}_{, 1}^{\mathrm{OR}}\right) / \partial \widehat{\gamma}_{, 1}^{\mathrm{OR}} .
$$

Since $\partial L_{n}^{\mathrm{OR}}\left(\widehat{\gamma}_{, 1}^{\mathrm{OR}}\right) / \partial \widehat{\gamma}_{, 1}^{\mathrm{OR}}=0$ together with (S.15), we have

$$
\partial L_{n}^{*}\left(\widehat{\gamma}_{, 1}^{\mathrm{OR}}, \gamma_{-1}\right) / \partial \widehat{\gamma}_{, 1}^{\mathrm{OR}}=O_{p}\left(n\left(J_{n}^{\mathcal{S}}\right)^{-1 / 2}\left(J_{n}^{\mathrm{ini}}\right)^{-r}\right) .
$$

By (S.16) and (S.17), we have

$$
\begin{aligned}
& \partial L_{n}^{*}\left(\widehat{\gamma}_{, 1}^{\mathrm{OR}}, \widehat{\gamma}_{,-1}^{\mathrm{ini}}\right) / \partial \widehat{\gamma}_{, 1}^{\mathrm{OR}}-\partial L_{n}^{*}\left(\widehat{\gamma}_{, 1}^{\mathrm{OR}}, \boldsymbol{\gamma}_{-1}\right) / \partial \widehat{\gamma}_{, 1}^{\mathrm{OR}} \\
= & \partial L_{n}^{\mathcal{S}}\left(\widehat{\gamma}_{, 1}^{\mathrm{OR}}\right) / \partial \widehat{\gamma}_{, 1}^{\mathrm{OR}}+O_{p}\left(n\left(J_{n}^{\mathcal{S}}\right)^{-1 / 2}\left(J_{n}^{\mathrm{ini}}\right)^{-r}\right) .
\end{aligned}
$$

Therefore, the result (S.12) follows from the above result and (S.14). In the following we will prove (S.14).

By Taylor expansion,

$$
\begin{aligned}
& \partial L_{n}^{*}\left(\widehat{\boldsymbol{\gamma}}_{, 1}^{\mathrm{OR}}, \widehat{\boldsymbol{\gamma}}_{,-1}^{\mathrm{ini}}\right) / \partial \widehat{\boldsymbol{\gamma}}_{, 1}^{\mathrm{OR}}-\partial L_{n}^{*}\left(\widehat{\boldsymbol{\gamma}}_{, 1}^{\mathrm{OR}}, \boldsymbol{\gamma}_{-1}\right) / \partial \widehat{\boldsymbol{\gamma}}_{, 1}^{\mathrm{OR}} \\
= & \left\{\partial^{2} L_{n}^{*}\left(\widehat{\boldsymbol{\gamma}}_{, 1}^{\mathrm{OR}}, \widetilde{\boldsymbol{\gamma}}_{-1}\right) / \partial \widehat{\boldsymbol{\gamma}}_{, 1}^{\mathrm{OR}} \partial \widetilde{\boldsymbol{\gamma}}_{-1}^{\mathrm{T}}\right\}\left(\widehat{\boldsymbol{\gamma}}_{,-1}^{\mathrm{ini}}-\boldsymbol{\gamma}_{-1}\right),
\end{aligned}
$$

where $\widetilde{\gamma}_{-1}=t \gamma_{-1}+(1-t) \widehat{\gamma}_{-1}$, for $t \in(0,1)$. Denote $\eta_{i}^{0}=\sum_{\ell=1}^{p}\left\{\alpha_{\ell 0}^{0}+\right.$ $\left.\sum_{k=1}^{d} \alpha_{\ell k}^{0}\left(X_{i k}\right)\right\} T_{i \ell}$, and $Z_{i j \ell, k}^{*}=B_{j, k}\left(X_{i k}\right) T_{i \ell}$ for $2 \leq k \leq d, Z_{i l, 0}^{*}=T_{i \ell}$, $Z_{i,-1}^{*}=\left\{\left(Z_{i l, 0}^{*}, Z_{i j \ell, k}^{*}, 1 \leq j \leq J_{n}^{\mathcal{S}}, 2 \leq k \leq d, \ell \in \widehat{I_{1}}\right)^{\mathrm{T}}\right\}$, and $Z_{i}^{*}=$ $\left\{\left(Z_{i l, 0}^{*}, Z_{i j \ell, k}^{*}, 1 \leq j \leq J_{n}^{\mathcal{S}}, 1 \leq k \leq d, \ell \in \widehat{I_{1}}\right)^{\mathrm{T}}\right\}$. Let $\epsilon_{i}=Y_{i}-g^{-1}\left(\eta_{i}\right)$, then

$$
\begin{aligned}
\widehat{\gamma}_{\widehat{I}_{1}}^{\text {ini }}-\gamma_{\widehat{I}_{1}}=\left\{n^{-1}\right. & \left.\sum_{i=1}^{n} q_{2}\left(\eta_{i}^{0}, Y_{i}\right) Z_{i}^{*} Z_{i}^{* \mathrm{~T}}\right\}^{-1} \\
& \times\left\{n^{-1} \sum_{i=1}^{n}\left\{Y_{i}-g^{-1}\left(\eta_{i}^{0}\right)\right\} \rho_{1}\left(\eta_{i}^{0}\right) Z_{i}^{*}\right\}\left\{1+o_{p}(1)\right\} .
\end{aligned}
$$

Following similar reasoning as the proof for (S.5), it can be shown that there exist constants $0<c_{1} \leq C_{1}<\infty$, such that with probability approaching 1 ,

$$
\begin{aligned}
c_{1} \mathbf{I}_{s^{*} d J_{n}^{s}} & \leq n^{-1} \sum_{i=1}^{n} q_{2}\left(\eta_{i}^{0}, Y_{i}\right) Z_{i}^{*} Z_{i}^{* \mathrm{~T}} \leq C_{1} \mathbf{I}_{s^{*} d J_{n}^{s}} \\
C_{1}^{-1} \mathbf{I}_{s^{*} d J_{n}^{s}} & \leq\left(n^{-1} \sum_{i=1}^{n} q_{2}\left(\eta_{i}^{0}, Y_{i}\right) Z_{i}^{*} Z_{i}^{* \mathrm{~T}}\right)^{-1} \leq c_{1}^{-1} \mathbf{I}_{s^{*} d J_{n}^{s}} .
\end{aligned}
$$

By $(\mathrm{S} .13) \partial^{2} L_{n}^{*}\left(\widehat{\gamma}_{, 1}^{\mathrm{OR}}, \widetilde{\gamma}_{-1}\right) / \partial \widehat{\gamma}_{, 1}^{\mathrm{OR}} \partial \widetilde{\gamma}_{-1}^{\mathrm{T}}=\sum_{i=1}^{n} q_{2}\left(\eta_{i}^{0}, Y_{i}\right) Z_{i, 1} Z_{i,-1}^{* \mathrm{~T}}$. Thus with probability approaching 1 ,

$$
\left\{\partial^{2} L_{n}^{*}\left(\widehat{\boldsymbol{\gamma}}_{, 1}^{\mathrm{OR}}, \widetilde{\boldsymbol{\gamma}}_{-1}\right) / \partial \widehat{\boldsymbol{\gamma}}_{, 1}^{\mathrm{OR}} \partial \widetilde{\boldsymbol{\gamma}}_{-1}^{\mathrm{T}}\right\}\left(\widehat{\boldsymbol{\gamma}}_{-1}^{\mathrm{ini}}-\boldsymbol{\gamma}_{-1}\right) \asymp \zeta_{1}+\zeta_{2},
$$


where

$$
\begin{aligned}
\zeta_{1} & =n^{-1}\left\{\sum_{i=1}^{n} q_{2}\left(\eta_{i}^{0}, Y_{i}\right) Z_{i, 1} Z_{i,-1}^{* \mathrm{~T}}\right\}\left\{\sum_{i=1}^{n}\left\{g^{-1}\left(\eta_{i}\right)-g^{-1}\left(\eta_{i}^{0}\right)\right\} \rho_{1}\left(\eta_{i}^{0}\right) Z_{i,-1}^{*}\right\}, \\
\zeta_{2} & =n^{-1}\left\{\sum_{i=1}^{n} q_{2}\left(\eta_{i}^{0}, Y_{i}\right) Z_{i, 1} Z_{i,-1}^{* \mathrm{~T}}\right\}\left\{\sum_{i=1}^{n} \epsilon_{i} \rho_{1}\left(\eta_{i}^{0}\right) Z_{i,-1}^{*}\right\} .
\end{aligned}
$$

Now, $\zeta_{1} \asymp\left\{n^{-1} \sum_{i, i^{\prime}} Z_{i, 1} Z_{i,-1}^{* \mathrm{~T}} Z_{i^{\prime},-1}^{*}\right\} O\left(N^{-r}\right)=\left(\zeta_{11}+\zeta_{12}\right) O\left(N^{-r}\right)$, where

$$
\begin{aligned}
\zeta_{11}= & n^{-1} \sum_{i} Z_{i, 1} Z_{i,-1}^{* \mathrm{~T}} Z_{i,-1}^{*}=\left(\zeta_{11, j^{\prime} \ell^{\prime}}\right)_{1 \leq j^{\prime} \leq J_{n}^{\delta}, \ell^{\prime} \in \widehat{I}_{1}}, \\
\zeta_{11, j^{\prime} \ell^{\prime}}= & n^{-1} \sum_{i} \sum_{j, k \neq 1, \ell} B_{j^{\prime}, 1}^{\mathcal{S}}\left(X_{i 1}\right) B_{j, k}^{2}\left(X_{i k}\right) T_{i \ell}^{2} T_{i \ell^{\prime}} \\
& +n^{-1} \sum_{i} \sum_{\ell} B_{j^{\prime}, 1}^{\mathcal{S}}\left(X_{i 1}\right) T_{i \ell}^{2} T_{i \ell^{\prime}}
\end{aligned}
$$

and

$$
\begin{aligned}
\zeta_{12}= & n^{-1} \sum_{i \neq i^{\prime}} Z_{i, 1} Z_{i,-1}^{* \mathrm{~T}} Z_{i^{\prime},-1}^{*}=\left(\zeta_{12, j^{\prime} l^{\prime}}\right)_{1 \leq j^{\prime} \leq J_{n}^{S}, \ell^{\prime} \in \widehat{I}_{1}}, \\
\zeta_{12, j^{\prime} l^{\prime}}= & n^{-1} \sum_{i \neq i^{\prime}} \sum_{j, k \neq 1, \ell} B_{j^{\prime}, 1}^{\mathcal{S}}\left(X_{i 1}\right) B_{j, k}\left(X_{i k}\right) B_{j, k}\left(X_{i^{\prime} k}\right) T_{i \ell} T_{i \ell^{\prime}} T_{i^{\prime} \ell} \\
& +n^{-1} \sum_{i \neq i^{\prime}} \sum_{\ell} B_{j^{\prime}, 1}^{\mathcal{S}}\left(X_{i 1}\right) T_{i \ell} T_{i \ell^{\prime}} T_{i^{\prime} \ell} .
\end{aligned}
$$

Since $E\left(B_{j^{\prime}, 1}^{\delta}\left(X_{i 1}\right) B_{j, k}^{2}\left(X_{i k}\right) T_{i \ell}^{2} T_{i l^{\prime}}\right) \asymp\left(J_{n}^{\delta}\right)^{-1 / 2}, E\left(B_{j^{\prime}, 1}^{\S}\left(X_{i 1}\right) T_{i \ell}^{2} T_{i l^{\prime}}\right) \asymp\left(J_{n}^{\S}\right)^{-1 / 2}$, $E\left\{B_{j^{\prime}, 1}^{\mathcal{S}}\left(X_{i 1}\right) B_{j, k}\left(X_{i k}\right) B_{j, k}\left(X_{i^{\prime} k}\right) T_{i \ell} T_{i l^{\prime}} T_{i^{\prime} \ell}\right\} \asymp\left(J_{n}^{\mathcal{S}}\right)^{-1 / 2}\left(J_{n}^{\text {ini }}\right)^{-1}$, and $E\left\{B_{j^{\prime}, 1}^{\mathcal{S}}\left(X_{i 1}\right) T_{i \ell} T_{i l^{\prime}} T_{i^{\prime} \ell}\right\} \asymp\left(J_{n}^{\mathcal{S}}\right)^{-1 / 2}$. It can be proved by Bernstein's inequality that $\left\|\zeta_{11}\right\|_{\infty}=O_{a . s .}\left\{J_{n}\left(J_{n}^{\mathcal{S}}\right)^{-1 / 2}\right\},\left\|\zeta_{12}\right\|_{\infty}=O_{a . s .}\left\{n\left(J_{n}^{\mathcal{S}}\right)^{-1 / 2}\right\}$. Therefore

$$
\left\|\zeta_{1}\right\|_{\infty}=O_{a . s .}\left\{n\left(J_{n}^{\mathcal{S}}\right)^{-1 / 2} J_{n}^{-r}\right\} .
$$

Denote $\boldsymbol{\Omega}=\left(\omega_{j \ell, 0 \ell^{\prime}}, \omega_{j \ell, j^{\prime} k \ell^{\prime}}\right)=n^{-1}\left\{\sum_{i=1}^{n} q_{2}\left(\eta_{i}^{0}, Y_{i}\right) Z_{i, 1} Z_{i,-1}^{* \mathrm{~T}}\right\}$ and $\mu_{\boldsymbol{\Omega}}=$ $E(\boldsymbol{\Omega})$. Then $E\left(\omega_{j \ell, j^{\prime} k \ell^{\prime}}\right) \asymp\left(J_{n}^{\mathcal{S}} J_{n}\right)^{-1 / 2}$ and $E\left(\omega_{j \ell, 0 \ell^{\prime}}\right) \asymp\left(J_{n}^{\mathcal{S}}\right)^{-1 / 2}$. It can be proved by Bernstein's inequality that $\sup _{j, j^{\prime}, \ell, \ell^{\prime}, k}\left|\omega_{j \ell, j^{\prime} k \ell^{\prime}}-E\left(\omega_{j \ell, j^{\prime} k \ell^{\prime}}\right)\right|=$ $O_{a . s .}\left\{(\log n / n)^{1 / 2}\right\}$, and that $\sup _{j, \ell, \ell^{\prime}}\left|\omega_{j \ell, 0 \ell^{\prime}}-E\left(\omega_{j \ell, 0 \ell^{\prime}}\right)\right|=O_{a . s .}\left\{\left(J_{n}^{S} \log n / n\right)^{1 / 2}\right\}$. Define $\widetilde{\zeta}_{2}=\left(\widetilde{\zeta}_{2, j^{\prime} \ell^{\prime}}\right)_{1 \leq j^{\prime} \leq J_{n}^{s}, \ell^{\prime} \in \widehat{I}_{1}}=\mu_{\boldsymbol{\Omega}}\left\{\sum_{i=1}^{n} \epsilon_{i} \rho_{1}\left(\eta_{i}^{0}\right) Z_{i,-1}^{*}\right\}$, so that $\widetilde{\zeta}_{2, j^{\prime} \ell^{\prime}} \asymp$ $\widetilde{\zeta}_{12, j^{\prime} \ell^{\prime}}+\widetilde{\zeta}_{22, j^{\prime} \ell^{\prime}}$, where

$$
\begin{aligned}
\widetilde{\zeta}_{12, j^{\prime} \ell^{\prime}} & =\sum_{i=1}^{n} \sum_{j, k \neq 1, \ell} E\left(\omega_{j^{\prime} \ell^{\prime}, j k \ell}\right) B_{j, k}\left(X_{i k}\right) T_{i \ell} \epsilon_{i} \\
\widetilde{\zeta}_{22, j^{\prime} \ell^{\prime}} & =\sum_{i=1}^{n} \sum_{\ell} E\left(\omega_{j^{\prime} \ell^{\prime}, 0 \ell}\right) T_{i \ell} \epsilon_{i} .
\end{aligned}
$$

By the weak law of large numbers, $\sup _{j^{\prime}, \ell^{\prime}}\left|\widetilde{\zeta}_{22, j^{\prime} \ell^{\prime}}\right|=\left(\sqrt{n / J_{n}^{\mathcal{S}}}\right)$. Let $\xi_{i}=$ $\sum_{j, k \neq 1, \ell} E\left(\omega_{j^{\prime} \ell^{\prime}, j k \ell}\right) B_{j, k}\left(X_{i k}\right) T_{i \ell} \epsilon_{i}$, then $E\left(\xi_{i}\right)=0, \operatorname{var}\left(\xi_{i}\right) \asymp\left(J_{n}^{\mathcal{S}}\right)^{-1}$. It can 
be proved by Bernstein's inequality that $\sup _{j^{\prime}, \ell^{\prime}}\left|\widetilde{\zeta}_{12, j^{\prime} \ell^{\prime}}\right|=O_{a . s .}\left(\sqrt{n \log n / J_{n}^{\delta}}\right)$. Hence

$$
\left\|\zeta_{2}\right\|_{\infty}=O_{p}\left(\sqrt{n \log n / J_{n}^{\mathcal{S}}}\right)
$$

Therefore, by (S.19), (S.20) and (S.21), we have

$$
\begin{aligned}
& \left\|\left\{\partial^{2} L_{n}^{*}\left(\widehat{\gamma}_{, 1}^{\mathrm{OR}}, \widetilde{\gamma}_{-1}\right) / \partial \widehat{\gamma}_{, 1}^{\mathrm{OR}} \partial \widetilde{\gamma}_{-1}^{\mathrm{T}}\right\}\left(\widehat{\gamma}_{-1}^{\mathrm{ini}}-\gamma_{-1}\right)\right\|_{\infty} \\
= & O_{p}\left\{n\left(J_{n}^{\S}\right)^{-1 / 2}\left(J_{n}^{\mathrm{ini}}\right)^{-r}+\sqrt{n \log n / J_{n}^{\S}}\right\} .
\end{aligned}
$$

The above result together with (S.18) implies

$$
\begin{aligned}
& \left\|\partial L_{n}^{*}\left(\widehat{\gamma}_{, 1}^{\mathrm{OR}}, \widehat{\gamma}_{,-1}^{\mathrm{ini}}\right) / \partial \widehat{\gamma}_{, 1}^{\mathrm{OR}}-\partial L_{n}^{*}\left(\widehat{\gamma}_{, 1}^{\mathrm{OR}}, \boldsymbol{\gamma}_{-1}\right) / \partial \widehat{\boldsymbol{\gamma}}_{, 1}^{\mathrm{OR}}\right\|_{\infty} \\
= & O_{p}\left\{n\left(J_{n}^{\mathcal{S}}\right)^{-1 / 2}\left(J_{n}^{\mathrm{ini}}\right)^{-r}+\sqrt{n \log n / J_{n}^{S}}\right\},
\end{aligned}
$$

so that (S.14) is proved.

S.1. Proof of (27). In the following, we will provide the proofs for the result (27). Let $\nabla L_{n}^{\mathcal{S}}\left(\boldsymbol{\gamma}_{, 1}\right)=\partial L_{n}^{\mathcal{S}}\left(\boldsymbol{\gamma}_{, 1}\right) / \partial \boldsymbol{\gamma}_{, 1}$ and $\nabla^{2} L_{n}^{\mathcal{S}}\left(\boldsymbol{\gamma}_{, 1}\right)=\partial^{2} L_{n}^{\mathcal{S}}\left(\boldsymbol{\gamma}_{, 1}\right) / \partial \boldsymbol{\gamma}_{, 1} \partial \boldsymbol{\gamma}_{, 1}^{\mathrm{T}}$. By Taylor expansion, $\widehat{\gamma}_{, 1}^{\mathcal{S}}-\widehat{\gamma}_{, 1}^{\mathrm{OR}}=-\left\{\nabla^{2} L_{n}^{\mathcal{S}}\left(\widetilde{\gamma}_{, 1}\right)\right\}^{-1} \nabla L_{n}^{\mathcal{S}}\left(\widehat{\gamma}_{, 1}^{\mathrm{OR}}\right)$, where $\widetilde{\gamma}_{, 1}=$ $t \widehat{\gamma}_{, 1}^{\mathrm{OR}}+(1-t) \widehat{\gamma}_{, 1}^{\mathcal{S}}$. Denote

$$
\eta_{i}^{*}\left(\gamma_{, 1}\right)=\sum_{\ell \in \widehat{I}_{1}}\left\{\widehat{\gamma}_{\ell 0}^{\mathrm{ini}}+\sum_{k=2}^{d} B_{k}\left(X_{i k}\right)^{\mathrm{T}} \widehat{\gamma}_{\ell k}^{\mathrm{ini}}+B_{1}^{\mathcal{S}}\left(X_{i 1}\right)^{\mathrm{T}} \gamma_{\ell 1}\right\} T_{i \ell}
$$

where $\boldsymbol{\gamma}_{, 1}=\left(\boldsymbol{\gamma}_{\ell 1}^{\mathrm{T}}, \ell \in \widehat{I}_{1}\right)^{\mathrm{T}}$. Then $\nabla^{2} L_{n}^{\mathcal{S}}\left(\widetilde{\gamma}_{, 1}\right)=\sum_{i=1}^{n} Z_{i, 1} Z_{i, 1}^{\mathrm{T}} q_{2}\left(\eta_{i}^{*}\left(\widetilde{\gamma}_{, 1}\right), Y_{i}\right)$. Following similar reasoning as the proof for (S.5), it can be proved that for any $\vartheta \in R^{s^{*} J_{n}^{\delta}}$, there exist constants $0<c_{1} \leq C_{1}<\infty$, such that with probability approaching $1, c_{1} n \leq\left\|\nabla^{2} L_{n}^{\mathcal{S}}\left(\widetilde{\gamma}_{, 1}\right)\right\|_{2} \leq C_{1} n$, and thus

$$
\left(c_{1} n\right)^{-1} \leq\left\|\left\{\nabla^{2} L_{n}^{\mathcal{S}}\left(\widetilde{\gamma}_{, 1}\right)\right\}^{-1}\right\|_{2} \leq\left(c_{1} n\right)^{-1} .
$$

By Theorem 13.4.3 of DeVore and Lorentz (1993), one has $\left\|\left\{\nabla^{2} L_{n}^{\mathcal{S}}\left(\widetilde{\gamma}_{, 1}\right)\right\}^{-1}\right\|_{\infty}=$ $O_{a . s .}\left(n^{-1}\right)$. Therefore, by (S.12) and (S.22),

$$
\begin{aligned}
\left\|\widehat{\gamma}_{, 1}^{\mathcal{S}}-\partial \widehat{\gamma}_{, 1}^{\mathrm{OR}}\right\|_{\infty} & \leq\left\|\left\{\nabla^{2} L_{n}^{\mathcal{S}}\left(\widetilde{\gamma}_{, 1}\right)\right\}^{-1}\right\|_{\infty}\left\|\nabla L_{n}^{\mathcal{S}}\left(\widehat{\gamma}_{, 1}^{\mathrm{OR}}\right)\right\|_{\infty} \\
& =O_{p}\left(\sqrt{\log n /\left(J_{n}^{\mathcal{S}} n\right)}+\left(J_{n}^{\mathcal{S}}\right)^{-1 / 2}\left(J_{n}^{\mathrm{ini}}\right)^{-r}\right)
\end{aligned}
$$




\section{References.}

Bosq, D. (1998). Nonparametric Statistics for Stochastic Processes. Springer-Verlag, New York.

DeVore, R. A. and Lorentz, G. G. (1993). Constructive Approximation. SpringerVerlag, Berlin.

Gangwisch, J. E., Malaspina, D., Boden-Albala, B. and Heymsfield, S. B. (2005). Inadequate sleep as a risk factor for obesity: analyses of the NHANES I. Sleep 28 1289-1296.

HUAng, J., Horowitz, J. and WeI, F. (2010). Variable selection in nonparametric additive models. Annals of Statistics 38 2282-2313.

LiAn, H. (2012). Variable selection for high-dimensional generalized varying-coefficient models. Statistica Sinica 22 1563-1588.

Thompson, C. L., Larkin, E. K., Patel, S., Berger, N. A., Redline, S. and Li, L. (2011). Short duration of sleep increases risk of colorectal adenoma. Cancer 117 841847.

Tibshirani, R., Bien, J., Friedman, J., Hastie, T., Simon, N., Taylor, R. and TibshiRANI, R. (2010). Strong rules for discarding predictors in lasso-type problems Technical Report, Standford University.

Wang, L., Liu, X., Liang, H. and Carroll, R. (2011). Estimation and variable selection for generalized additive partial linear models. Annals of Statistics 39 1827-1851.

SHuJie MA

Department of Statistics

University of CALIFornia at Riverside

RIVERSIDE, CA 92521, USA

E-MAIL: shujie.ma@ucr.edu

HuA Liang

Department of Statistics

George Washington University

WAShington, D.C. 20052, USA

E-MAIL: hliang@gwu.edu
Raymond J. CARRoll

Department of Statistics

TEXAS A\&M UNIVERSITY

College Station, TX 77843, USA

and School of Mathematical Sciences

University of TeChNOLOGY

Sydney, BRoAdWAY NSW 2007

E-MAIL: carroll@stat.tamu.edu

Shizhong XU

Center for Plant Cell Biology

University of CALIFORNia AT Riverside

Riverside, CA 92521, USA

E-MAIL: shizhong.xu@ucr.edu 